\title{
Professional Diploma of Family Medicine in Egypt and Sudan: Face-to-Face Versus Synchronous Distant Learning
}

\author{
Marwa Mostafa Ahmed*, Ghada Mahmoud Khafajy, Nagwa Eid Saad \\ Department of Family Medicine, Cairo University, Cairo, Egypt
}

Email address:

marwamostafa@kasralainy.edu.eg (M. M. Ahmed)

\section{To cite this article:}

Marwa Mostafa Ahmed, Ghada Mahmoud Khafajy, Nagwa Eid Saad. Professional Diploma of Family Medicine in Egypt and Sudan: Faceto-Face Versus Synchronous Distant Learning. European Journal of Preventive Medicine. Vol. 3, No. 6, 2015, pp. 197-200. doi: 10.11648/j.ejpm.20150306.17

\begin{abstract}
Background: Videoconferencing technology reduces time and costs between remote locations, fill gaps in teaching services, enables meetings that would not be possible due to prohibitive travel costs, and improves access to learning Aim: To explore the effectiveness of the theoretical module of PDFM (Professional Diploma of Family Medicine) in Sudan delivered via distant learning compared to that of PDFM in Egypt delivered via conventional learning by comparing the achievement level in the post-theoretical-module exam and the results of the exam analysis. Methods: This comparative study was conducted from November 2014 to April 2015. In PDFM Egypt and Sudan, the theoretical part was delivered via face-to face learning and synchronous videoconference respectively. The same exam was used to assess candidates' knowledge in PDFM in Egypt and Sudan at the same time. Item analysis was done to obtain item and test statistics. Also, candidates' feedback was assessed using a Web-based questionnaire Results: Thirty-two candidates (84.2\%) passed the exam of PDFM in Egypt while 77 candidates (86.5\%) passed it in Sudan. The reliability co-efficient of the exam in Egypt and Sudan were 0.9188 and 0.8140 respectively. There was no significant difference between the discrimination index of the exam ( $p$ value $=0.074$ ), while there was significant difference between the difficulty index; $70 \%$ of the exam questions were considered easy in PDFM Sudan compared to $40 \%$ in PDFM Egypt. Conclusion: Synchronous videoconferencing can be used to expand educational capacity and international cooperation between academic institutions in developing countries, a particular priority in the growing field of Family Medicine.
\end{abstract}

Keywords: Distant Learning, Family Medicine, Face-to-Face Learning, Videoconferencing

\section{Introduction}

Distance learning programs range from independent study to more formal coursework offered by various asynchronous (email, Web) and synchronous (videoconferencing) technologies and may include "blended learning" approaches combining distance education with face-to-face instruction [1]. Previous research indicates that students taught at a distance usually have no significant learning differences from face to- face students [2].

Reasons mentioned by medical students for preferring web tutorials rather than traditional lecture-based classes included accessibility, ease of use, freedom of navigation and the possibility of repeat practice. Despite its advantages, the primary drawbacks to asynchronous online learning are technical issues and the lack of interactions among students and the instructor. These findings may be the reason that integration of electronic education with classroom problem solving sessions or other face to face activities is necessary (3).

Videoconference-based education is the use of communication technology, such as phone lines (integrated services digital network [ISDN]) or the Internet (Internet protocol [IP]) to provide education from a central site to remote distant sites using live interactive audio-video communication. There are a wide range of video systems in use and they vary in terms of costs and resolution of video images [1]. Videoconferencing has become a wellestablished distance-learning technique for medical education, being used across a wide range of health disciplines including, pharmacology [3], nursing [4] and psychiatry [5].

Videoconferencing experience is considered to be close to face-to-face experience for students in remote locations, 
where students indicated presenter personality, interactivity, and teaching style were more important than point of origin. Also students engaged in distance learning via videoconferencing thought that direct contact with international experts was considered a unique opportunity and that the use of this medium created a sense of intimacy and rapport despite the challenges posed by distance. However the primary concern was the students' perception that the videoconference tools were barrier to their interaction with the instructor [6, 7].

Videoconferencing technology reduces time and costs between remote locations, fill gaps in teaching services, increases training productivity, enables meetings that would not be possible due to prohibitive travel costs, and improves access to learning [1].

Since, implementation of family medicine in Africa faces many challenges including limited funding, focusing on hospital care, poor training capacity, and drainage of doctors towards rich or more developed countries. Hence, the Arab Institute of Continuing Professional Development (AICPD) introduced the professional diploma in family medicine (PDFM) to face the shortage in family practitioners in Egypt and Sudan. In order to reduce the travel costs and improve access to learning, the theoretical module of PDFM in Sudan was done via synchronous videoconferencing. This study is an attempt to explore the effectiveness of the theoretical module of PDFM in Sudan delivered via distant learning compared to that of PDFM in Egypt delivered via conventional learning by comparing the achievement level in the progression exam and the results of the exam analysis.

\section{Methods}

\subsection{Study Design}

This comparative study was conducted from November 2014 to April 2015

\subsection{Study Participants}

Candidates enrolled in the PDFM (patch 7) in Egypt and candidates enrolled in PDFM (patch 3) in Sudan.

\subsection{PDFM Contents}

The duration of the PDFM is one year and it is composed of three modules:

1. Theoretical background of family medicine: 40 days (2days / 20 weeks). Each day consists of 6-hour lecture including half an hour break. Lectures includes common and important topics in family medicine, internal medicine, pediatrics, obstetrics and gynecology, surgery, psychiatry, dermatology, ophthalmology, ear, nose and thorax and pharmacology.

2. Practical training in family medicine

3. Clinical and field training in family medicine

In PDFM Sudan, the theoretical part and half of the lectures in practical part are delivered via synchronous videoconference (Distant learning). While the rest of the course content is delivered on site via face to face approach. On the other hand, the course content was delivered via face-to face learning (Conventional learning) in PDFM Egypt.

Both courses had the same learning objectives and content comprised of the same teaching presentation and handouts, planned and presented by the same Egyptian lecturers. The sequence of PowerPoint slides and video in PDFM Sudan were shown in the same order as the PDFM Egypt and the candidates completed both courses in the same time frame.

\subsection{Outcome Assessment}

The same post-theoretical-module exam was used to assess candidates' knowledge in PDFM in Egypt and Sudan at the same time. The exam was constructed by the head of the PDFM scientific council. The questions were selected from questions pool constructed by members of the scientific council. The exam included 50 EMQs, all of these EMQs are problem focused referring to realistic cases. For each EMQ, a point value of 1 was assigned, for a total possible score of 50 points. Also, candidates' feedback was assessed using a Webbased questionnaire. Each question was scored on a 5-point Likert scale.

\subsection{Item Analysis of Exam}

Item analysis was done using Classical Test Theory to obtain item and test statistics. Item statistics include difficulty index and discrimination index.

Difficulty index describes the percentage of students who correctly answered a test item. The cut-off points to evaluate the difficulty index of MCQs were: $>70 \%$ (easy); $20-70 \%$ (moderate) $;<20 \%$ (difficult). Moderate difficulty items (20$70 \%$ ) in a test have better discriminating ability [8]. Discriminating index is the ability of a test item to discriminate between high and low examinee scorers. Higher discriminating index in an item indicate greater discriminating capability of that item. The cut-off values for (DI) are as follow: $>0.15$ (discriminating index) and $\leq 0.15$ (non-discriminating index) [9].

Test reliability was measured by cronbach alpha. It refers to the extent to which the test is likely to produce a consistent score. It ranges in value from zero (no reliability) to 1.00 (perfect reliability) [8].

\subsection{Statistical Analysis}

The collected data were statistically analyzed using statistical Package for Social Sciences (SPSS) version 16. Data were statistically described in terms of Mean, \pm Standard Deviation ( \pm SD) or Frequencies (Number of cases) and percentages when appropriate. The difference in the mean was assessed using independent $t$ test. For comparing categorical data, Chi square $\left(\mathrm{X}^{2}\right)$ test was performed. Fisher Exact test was used instead when the expected frequency is less than 5. P value less than 0.05 was considered statistically. 


\section{Results}

Table 1. Test statistics of the PDFM Egypt and PDFM Sudan.

\begin{tabular}{lll}
\hline & PDFM Egypt & PDFM Sudan \\
\hline Number of candidates & 38 & 89 \\
Number of passed candidates & 32 & 77 \\
Mean score & $36 \pm 6.3$ & $37.2 \pm 6.4$ \\
Reliability coefficient & 0.9188 & 0.8140 \\
\hline
\end{tabular}

Table (1) shows that 32 candidates $(84.2 \%)$ passed the post-theoretical module exam of PDFM in Egypt while 77 candidates $(86.5 \%)$ passed it in Sudan. There was no significant difference between the mean of the score of both exams ( $p$ value $=0.334$ ). The results of the exam of PDFM in both setting were analyzed separately. The reliability coefficients of the exam in Egypt and Sudan were 0.9188 and 0.8140 respectively.

Table 2. Comparison between difficulty and discrimination index of the PDFM exam in Egypt and Sudan.

\begin{tabular}{|c|c|c|c|c|c|c|}
\hline \multirow[t]{2}{*}{ Item analysis } & & \multicolumn{2}{|c|}{$\begin{array}{l}\text { PDFM } \\
\text { Egypt }\end{array}$} & \multicolumn{2}{|c|}{$\begin{array}{l}\text { PDFM } \\
\text { Sudan } \\
\end{array}$} & \multirow{2}{*}{$\begin{array}{l}\mathbf{P} \\
\text { value* }\end{array}$} \\
\hline & & no & $\%$ & no & $\%$ & \\
\hline \multirow{2}{*}{$\begin{array}{l}\text { Discrimination } \\
\text { Index(DI) }\end{array}$} & $\mathrm{DI}(>0.15)$ & 6 & 12 & 13 & 26 & \multirow[b]{2}{*}{0.074} \\
\hline & $\begin{array}{l}\text { Non-DI } \\
(\leq 0.15)\end{array}$ & 44 & 88 & 37 & 74 & \\
\hline \multirow{3}{*}{$\begin{array}{l}\text { Difficulty } \\
\text { Index }\end{array}$} & $\begin{array}{l}\text { Difficult } \\
(<20 \%)\end{array}$ & 4 & 8 & 1 & 2 & \multirow{3}{*}{0.009} \\
\hline & $\begin{array}{l}\text { Moderated } \\
(20-70 \%)\end{array}$ & 26 & 52 & 14 & 28 & \\
\hline & $\begin{array}{l}\text { Easy } \\
(>70 \%)\end{array}$ & 20 & 40 & 35 & 70 & \\
\hline
\end{tabular}

Table (2) shows that there was no significant difference between the discrimination index of the exam ( $p$ value $=0.074$ ), while there was significant difference between the difficulty index of the exam ( $p$ value $=0.009$ ) in PDFM Egypt and Sudan.

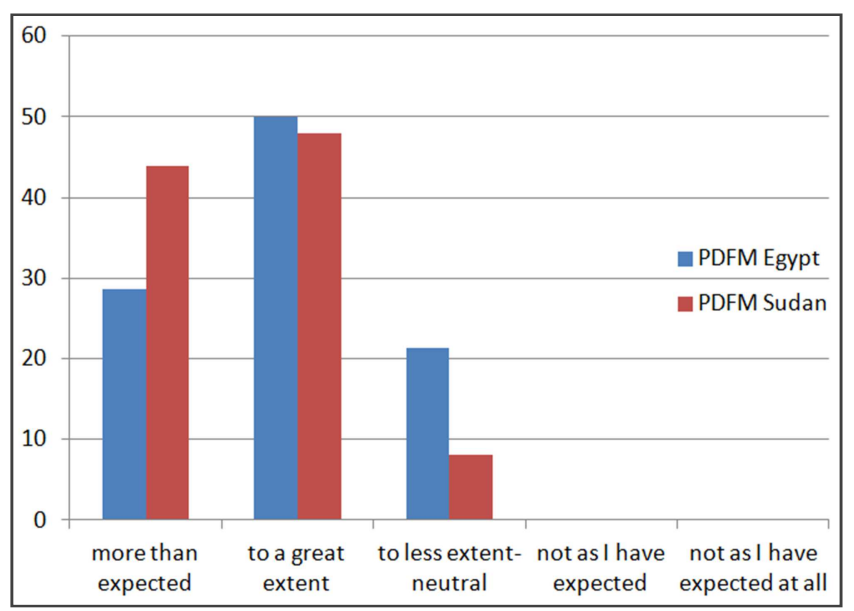

Figure 1. Candidates' response to the extent the course met their expectation.

The web-based questionnaire was returned by 14 candidates (response rate 41\%) from PDFM Egypt and 25 candidates (response rate 28\%) from PDFM Sudan. Their responses are shown in figures (1-3). In PDFM Sudan and Egypt, $44 \%$ and $28.6 \%$ of the candidates (who returned the questionnaire) stated that the course was better than they have expected. While $68 \%$ of the candidates in PDFM Sudan and $21.4 \%$ of the candidates in PDFM Egypt strongly agreed that the course covered all the aspects of family practice. Moreover, $76 \%$ of candidates in PDFM Sudan and $21.4 \%$ of the candidates in PDFM Egypt thought that the course instructors were excellent.

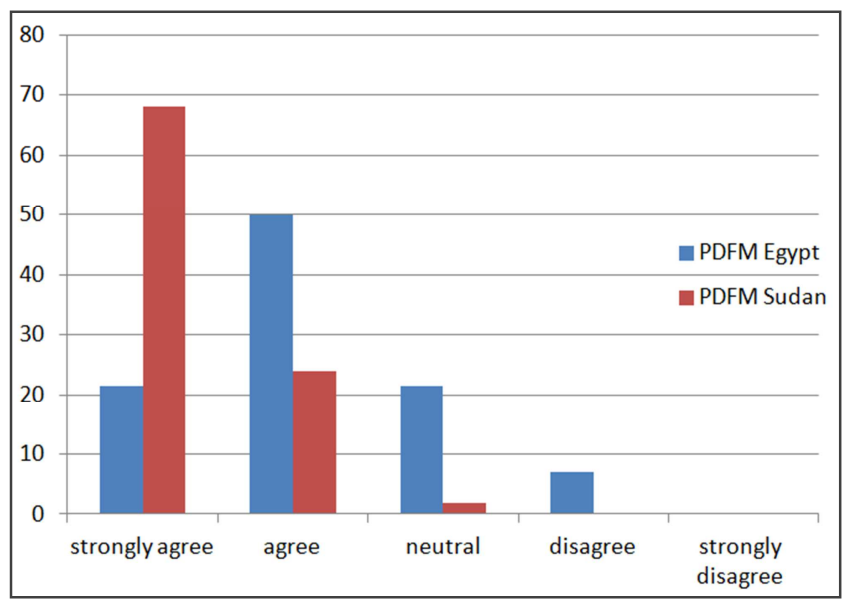

Figure 2. Candidates' response to the whether the course covered all the aspect of family practice or not.

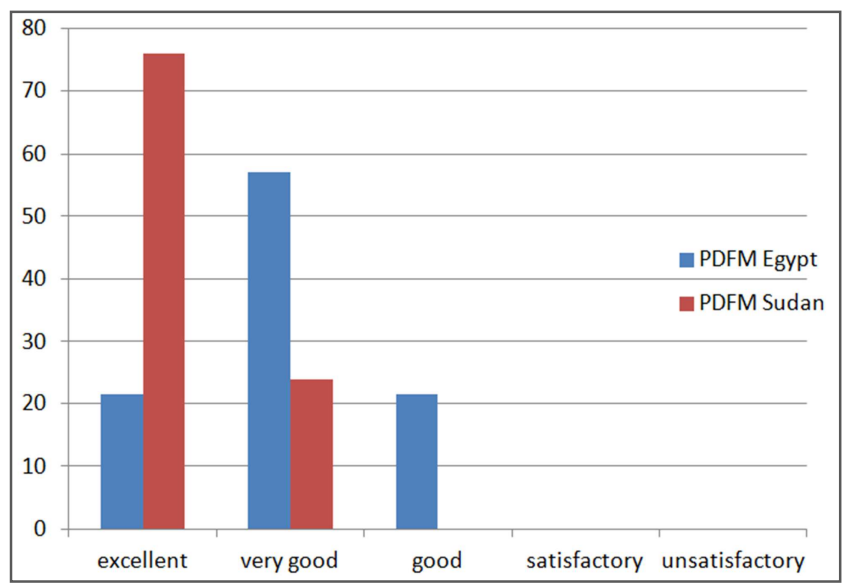

Figure 3. Candidates' overall opinion regarding course instructors.

\section{Discussion}

In our study, synchronous videoconferencing has proved to be an effective method in delivering family medicine education and bridging the geographical divide across countries. The percentage of candidates who has passed the post-theoretical-module exam was nearly the same in PDFM Egypt delivered via face-to-face learning and PDFM Sudan delivered by synchronous videoconferencing $(84.2 \%$ versus $86.5 \%$ ). Also, there was no significant difference between the mean of the exam score in both setting. This finding is consistent with the results of a review done by Augestad and 
Lindsetmo in 2009 [10], where synchronous videoconferencing has proven to be an effective educational tool for surgical education in remote areas. Also, in a systematic review done by Chipps and his colleagues in 2012 [4], four studies shows no inferiority of videoconferencebased education to other modes in scoring knowledge and one study indicated an increase in knowledge (in terms of tests taken by the participants) in videoconference-based education.

Since, the presence of confounding variables is a methodological problem for educational evaluation, therefore we tried to standardize the exam that was used to compare between both cohorts. Also, the psychometric analysis of the exam ensured the consistency and homogeneity of the exam in both settings; the reliability coefficients of the exam in Egypt and Sudan were 0.9188 and 0.8140 respectively. Moreover, there was no significant difference between the discriminating indexes in both setting. This indicates that the constructed exam had the same ability in discriminating between high and low candidates' scorers in both setting. Surprisingly, there was significance difference in the difficulty index of the exam, $70 \%$ of the exam questions were considered easy (difficulty level $>70 \%$ ) in PDFM Sudan compared to $40 \%$ of exam questions in PDFM Egypt.

One of the most important drawbacks of web-based learning is the distant nature of interactions between students and instructors which ended up by the candidates feeling isolated from the instructors [11]. This was not the case in PDFM Sudan, although the course content was delivered by videoconferencing, candidates-instructors interaction was maintained and every lecture ended by solving problembased questions or sharing in role play.

Although the response of the candidates to the web based questionnaire was low (response rates of PDFM Egypt and PDFM Sudan were $41 \%$ and $28 \%$ respectively). Completing the course and entering the exam can be considered as an indirect indication of candidates' satisfaction.

Strengths and limitations

This is the first study of which we are aware to directly compare conventional and distant learning in the field of family medicine in Egypt and Sudan. One of the limitations of this study is the limited control of potentially confounding variables of the two cohorts. While this limitation can be controlled using a randomized design, in our study it was not applicable to randomize candidates to one of the two routes.

\section{Conclusion}

Achievement level was comparable between candidates of PDFM Egypt (delivered via conventional learning) and Sudan (delivered via distant learning). Synchronous videoconferencing can be used to expand educational capacity and international cooperation between academic institutions in developing countries, a particular priority in the growing field of Family Medicine.

\section{Authors' Contributions}

All authors contributed fully in formatting the research proposal, data collection and analysis, as well as the manuscript editing and submission

\section{References}

[1] Coventry L. Video Conferencing in Higher Education. Institute for computer-based learning. Heriot Watt University. $\begin{array}{lll}\text { Retrieved } & 10 / 7 / 2015 & \text { from }\end{array}$ http://www.agocg.ac.uk/reports/mmedia/video3/video3.pdf

[2] Khatony A, Nayery ND, Ahmadi F, Haghani H, VehvilainenJulkunen K. The effectiveness of web-based and face-to-face continuing education methods on nurses' knowledge about AIDS: a comparative study. BMC Medical Education 2009, 9: 41 doi: 10.1186/1472-6920-9-41.

[3] Moridani M: Asynchronous video streaming vs. synchronous videoconferencing for teaching a pharmacogenetic pharmacotherapy course. Am J Pharma Educ 2007, 71(1): 16.

[4] Chipps J, Brysiewicz P, Mars M. A Systematic Review of the Effectiveness of Videoconference-Based Tele-Education for Medical and Nursing Education. Worldviews on EvidenceBased Nursing 2012: doi: 10.1111/j.1741-6787.2012.00241.x.

[5] Chipps J, Ramlall S, Mars M.Videoconference-based education for psychiatry registrars at the University of KwaZulu-Natal, South Africa. Afr J Psychiatry 2012; 15:248254. doi: 10.4314/ajpsy.v15i4.32.

[6] Ekblad S, Manicavasagar V, Silove D, Bäärnhielm S, Reczycki M, Mollica R, Coello M. The Use of International Videoconferencing as a Strategy for Teaching Medical Students about Transcultural Psychiatry. Transculture psychiatry 2004. Vol 41(1): 120-129. DOI: 10.1177 / 1363461504041357.

[7] Doggett MA. The Videoconferencing Classroom: What Do Students Think? Journal of Industrial Teacher Education 2008; 44(4): 29-41.

[8] Understanding item analysis reports, University of Washington,

(http://www.washington.edu/oea/services/scanning_scoring/sc oring/item_analysis.html(2005), Date of access: 4/7/2015).

[9] Gajjar S., Sharma R., Kumar P., and Rana M. Item and Test Analysis to Identify Quality Multiple Choice Questions (MCQs) from an Assessment of Medical Students of Ahmedabad, Gujarat. Indian J Community Med. 2014; 39(1): 17-20.

[10] Augestad K, Lindsetmo R. Overcoming Distance: VideoConferencing as a Clinical and Educational Tool Among Surgeons. World J Surg 2009; 33: 1356-1365. DOI 10.1007/s00268-009-0036-0.

[11] McKimm J, Jollie C, Cantillon P. ABC of learning and teaching Web based learning. BMJ 2003; 326: 870-873. 